Original Paper http://ajol.info/index.php/ijbcs http://indexmedicus.afro.who.int

\title{
Ecologie alimentaire de Labeobarbus altianalis (Boulenger, 1900) du Bassin de la rivière Luhoho, en territoire de Kalehe (Sud-Kivu, R.D. Congo)
}

\author{
Gabriel MUKABO OKITO $^{1 *}$, Dieu-donné CIKWANINE KASIGWA ${ }^{2}$, \\ Jean-Claude $\mathrm{MICHA}^{2}$, Vénant NSHOMBO MUDERHWA ${ }^{3}$, Osée RWAKANA \\ RIBAKARE $^{1}$, Phanuel BIZURU NZIBONERA ${ }^{5}$, B. Georges MUHIRWA ${ }^{5}$, \\ Donald E. MBOBOCI SUKU ${ }^{6}$ et Pierrot MUDIMBE KALUBA ${ }^{6}$
}

\author{
${ }^{I}$ Section Eaux et Forêts, Institut Supérieur Agrovétérinaire et de Conservation de la nature (ISAVC/Minembwe, \\ RDC), BP 6295 Bujumbura, Burundi. \\ ${ }^{2}$ Département de Biologie, Université Officielle de Bukavu, B.P. 570 Bukavu/RDC. \\ ${ }^{3}$ Département de Biologie, Centre de Recherche en Hydrobiologie d'Uvira, CRH-Uvira, BP-73Uvira, RD \\ Congo. \\ ${ }^{4}$ Département de Biologie, Unité de Recherche en Ecologie Environnementale (URBE) Université de Namur, \\ BP-5000 Namur, Belgique. \\ ${ }^{5}$ Section Agrovétérinaire, Institut Supérieur Agrovétérinaire et de Conservation de la nature de Minembwe \\ (ISAVC/Minembwe, RDC), B.P. 6295 Bujumbura, Burundi. \\ ${ }^{6}$ Section Développement, Institut Supérieur Agrovétérinaire et de Conservation de la nature de Minembwe \\ (ISAVC/Minembwe, RDC), B.P. 6295 Bujumbura, Burundi. \\ *Auteur correspondant, E-mail : mgokito@gmail.com ; Tél. : (+243) 821270424
}

\section{RESUME}

Le présent article analyse l'écologie alimentaire du poisson (Labeobarbus altianalis) Cyprinidae de manière à ressortir la qualitative et quantitative du régime alimentaire de ce poisson en fonction de la taille ; la détermination de son rythme d'activités trophique et locomotrice et enfin établir la relation existant entre l'activité trophique et locomotrice de Labeobarbus altianalis dans les différent sites de capture. L'échantillonnage dans les affluents de la rivière Luhoho a été effectué au mois de mars et de juin 2014. Pour chaque sortie, des échantillons de poissons étaient récolté dans chaque affluent de la rivière Luhoho. Chaque fois l'échantillonnage se faisait le matin, midi et soir respectivement entre $6 \mathrm{~h}-9 \mathrm{~h}, 10 \mathrm{~h}-12 \mathrm{~h}$ et $14 \mathrm{~h}-18 \mathrm{~h}$. Les poissons étaient capturés par 2 pêcheurs utilisant chacun un filet épervier de $10 \mathrm{~mm}$ de maille d'entre-noeuds et de $2 \mathrm{~m}^{2}$ de surface attachés à une longue corde au milieu et entouré dans sa périphérie par des plombs. Le temps du prélèvement du filet variait entre 2 et 3 minutes. L'analyse de résultats permet de conclure que l'espèce Labeobarbus altianalis des affluents de la rivière Luhoho est un poisson phytoplanctonophage se nourrissant essentiellement des diatomées (Bacillariophycées), des Cyanophytes, des Chlorophytes et d'Euglenophytes dans tous les sites considérés et à tous les âges. Son activité locomotrice serait plus intense entre 6 heures et 18 heures à Tchinganda et Eke. Par contre, à Tchoko et Nyamunene, c'est entre 14 heures et 18 heures qu'il se déplace intensément. L'activité locomotrice de ce poisson serait liée à la recherche de la nourriture. Il existe un lien étroit entre l'activité locomotrice de ce poisson et son alimentation dans tous les quatre sites d'échantillonnage choisis.

(C) 2016 International Formulae Group. All rights reserved.

Mots clés : Biologie, régime alimentaire, cycle nycthermal, Labeobarbus altianalis, Luhoho, République Démocratique du Congo. 


\title{
Food ecology of Labeobarbus altianalis (Teleostei, Cyprinidae) (Boulenger 1900) of the Pond of the river Luhoho, on territory of Kalehe (Sud-Kivu, R.D. Congo)
}

\begin{abstract}
The present article analyzes the food ecology of the fish Labeobarbus altianalis Cyprinidae so as to take out again the qualitative and quantitative of the diet of this fish according to the size; the determination of its rhythm of trophic activities and locomotive To establish finally the existing relation enters the trophic activity and motive unit of Labeobarbus altianalis in different capture sites. The sampling in the tributaries of the river Luhoho was made in March and June, 2014. For every exit, samples of fishes were harvested in every tributary of the Luhoho River. Every time the sampling was made in the morning, noon and evening respectively between 6 at 9 am, 10 at 12 am and 14 at 6 pm. Fishes were captured by 2 fishermen using each a net sparrow hawk of $10 \mathrm{~mm}$ of stitch and $2 \mathrm{~m}^{2}$ of surface attached in a long rope in the middle and surrounded in its periphery by lead. The time of the taking of the net varied between 2 and 3 minutes. The analysis of results (profits) allows to conclude that the species Labeobarbus altianalis of the tributaries of the Luhoho River is a fish phytoplanctonophage feeding essentially on diatoma (Bacillariophycae), on Cyanophytes, on Chlorophytes and on Euglenophytes in all the considered sites and at all the ages. His locomotive activity would be more intense between 6 am and $6 \mathrm{pm}$ to Tchinganda and Eke. On the other hand, to Tchoko and Nyamunene, it is between $2 \mathrm{pm}$ and $6 \mathrm{pm}$ when he moves intensely. The locomotive activity of this fish would be bound in search of the food. There is a narrow link between the locomotive activity of this fish and the food in every four chosen sites of sampling.
\end{abstract}

(C) 2016 International Formulae Group. All rights reserved.

Keywords: Biology, Diet, nycthermal cycle, Labeobarbus altianalis, Luhoho, Democratic Republic of Congo.

\section{INTRODUCTION}

Les rivières font partie des zones humides abritant de nombreux habitats et diverses espèces dont certaines sont migratrices. Dans l'hémisphère Nord, des espèces telles que saumon, castors, loutres, écrivisses, invertébrés, plantes et algues y jouent des fonctions importantes. (Bouleau 2007).Actuellement, la rivière Luhoho et ses affluents constituent les écosystèmes aquatiques exploités dans ce milieu pour l'approvisionnement des populations en protéines animales. Faire un usage optimal des rivières requiert la compréhension de leur fonctionnement. Dans un premier temps, la connaissance de l'écologie des poissons qui peuplent cette rivière est d'une importance capitale. Malgré leur importance, ces écosystèmes ont été largement oubliés ces quatre dernières décennies par les limnologistes et la compréhension en conséquence de ces rivières par rapport aux lacs et aux autres écosystèmes aquatiques de la région reste faible. Depuis le $19^{\text {ème }}$ siècle, plusieurs études ont été conduites dans divers réservoirs africains (lacs et rivières), du Nord au Sud et de l'Est à l'Ouest du continent en vue d'améliorer la pêche commerciale tout en préservant les stocks exploités (Lalèyè et al., 2004 ; Ibala, 2010; Zango et al., 2016; Gangbe et al., 2016 ; Kadjo et al., 2016).

En effet, à l'Ouest de la partie nord du lac Kivu, précisément à Bunyakiri, se trouve une grande rivière ayant une richesse ichthyologique importante composée des poissons dont l'espèce représentative est Labeobarbus altianalis (Boulenger, 1900) (Téleostei, Cyprinidae). L'écologie de l'une des espèces appartenant à l'ancien genre que Labeobarbus altianalis, (Barbus kerstenii) est 
déjà connue au lac Kivu (Waasalo, 2003). Vu que les milieux de vie diffèrent (milieu lotique d'une part et milieu lentique d'autre part) il est probable que Labeobarbus altianalis (ex Barbus altianalis) de la rivière affiche un comportement différent à celui de $B$. kerstenii du lac malgré leur appartenance au même à la même famille. A part une étude sur l'inventaire systématique de poisson du bassin des rivières Lowa et Luhoho faisant étant de 18 genres comprenant chacun presque une espèce excepté le genre Labeo. Ces espèces sont Clarias sp., Parauchenoglanis punctatus, Pollimyrus sp., Oreochromis niloticus, Mastacembellus congicus, Labeo lukulae, L. macrostomus, 'Barbus' sp., Bryconaethiops boulengeri, Brycinus aff. poptae, Pareutropius debauwi, Bagrus bajad, Bathybagrus graueri, Mormyrus caballus, Myomyrus aff. macrops, Marcusenius sp., Raiamas sp., Micralestes humilis et Distichodus altus (Kisekelwa et al., 2013). Malgré l'abondance de Labeobarbus altianalis dans cet écosystème justifiant la pratique de la pêche dans ce milieu, aucune étude n'a jusque-là été consacrée au régime alimentaire des poissons de ces rivières en général et de Labeobarbus altianalis en particulier.

C'est dans le but d'apporter notre contribution à l'écologie alimentaire de Labeobarbus altianalis de cet écosystème que nous avons analysé la composition qualitative et quantitative du régime alimentaire de Labeobarbus altianalis du bassin de la rivière Luhoho, une rivière d'importance socioéconomique non négligeable qui doit par conséquent attirer l'attention de scientifique ; malheureusement l'homme, par sa démographie explosive et ses erreurs, perturbe ces écosystèmes naturels et exerce une pression énorme sur les espèces, par des prélèvements irrationnels, pour satisfaire ses besoins nutritionnels sans aucune connaissance préalable de leur écologie. Il y aurait même des espèces qui disparaissent sans avoir été connues par des scientifiques. Outre la composition du régime alimentaire, cette étude vise plus spécifiquement à déterminer la qualité et la quantité des aliments consommés par cette espèce et ce, en fonction de la taille; déterminer son rythme d'activités trophique et locomotrice et enfin, établir la relation existant entre l'activité trophique et locomotrice de ce poisson dans les différents sites de capture.

\section{MATERIELS ET METHODES \\ Zone d'étude}

Cette étude s'est déroulée en République Démocratique du Congo, province du Sud-Kivu, territoire de Kalehe en son poste administratif de Bunyakiri situé sur l'axe routier Bukavu-Kisangani à $77 \mathrm{~km}$ de la ville de Bukavu. Précisément dans les affluents de la rivière Luhoho entre $02^{\circ} 05^{\prime} 34.4$ " 'Latitude sud et $28^{\circ} 34^{\prime} 28.9^{\prime}$ ' Longitude Est. Large de 30 mètres avec une profondeur moyenne de plus ou moins 6 mètres, la rivière Luhoho prend sa source dans le Parc National de Kahuzi-Biega (en province du Sud-Kivu) et déverse ses eaux dans la rivière Lowa à Walikale, territoire du Nord-Kivu. Son fond est typique de toutes les grandes rivières: vase et sable mou dans les endroits calmes (rive convexe d'un tournant, cône d'alluvions d'affluents), des roches dans les rapides et au milieu du cours. Lelong des rives se voient également de nombreux troncs d'arbres immergés. Les fonds rocheux caractéristiques des eaux tumultueuses sont surtout répandus dans le haut Tchinganda, Nyamunene entre Bitale et Bulambika. Les fonds caillouteux qui appartiennent à des eaux calmes sont bien répandus entre 1200 et 900 mètres d'altitude notamment les rivières Eke et Tchoko.

Le climat de la zone d'étude est tropical. Par rapport à l'hiver, les étés ont beaucoup plus de pluie. D'après Köppen et Geiger, le climat y est classé Aw. En moyenne la température à Bunyakiri est de $26^{\circ} \mathrm{C}$. Sur 
l'année, la précipitation moyenne est de 981 $\mathrm{mm}$ (Kisekelwa et al., 2012).

\section{Période, sites et techniques d'échantillonnage}

La récolte d'échantillon pour cette étude a été réalisée de mars à juin 2014 dans les quatre sites affluents de la rivière Luhoho, à savoir les rivières Eke, Nyamunene, Tchoko et Tchinganda en diversifiant les heures de pêche. Pour chaque sortie, des échantillons de poissons étaient récolté dans chaque affluent de la rivière Luhoho. Chaque fois l'échantillonnage se faisait le matin, midi et soir respectivement entre $6 \mathrm{~h}-9 \mathrm{~h}, 10 \mathrm{~h}-12 \mathrm{~h}$ et 14h-18 heures. Les poissons étaient capturés par 2 pêcheurs utilisant chacun un filet épervier de $10 \mathrm{~mm}$ de maille d'entre-noeuds et de $2 \mathrm{~m}^{2}$ de surface attachés à une longue corde au milieu et entouré dans sa périphérie par des plombs. Lorsque le filet est convenablement lancé, la force centrifuge de plomb étale le filet à la surface de l'eau. Le bord lesté s'enfonce plus rapidement que le centre et l'épervier descend en formant un entonnoir au-dessus du poisson. Le temps du prélèvement du filet variait entre 2 et 3 minutes.

\section{Position systématique de l'espèce étudiée : \\ Labeobarbus altianalis \\ Noms vernaculaires}

Les autochtones recourent à de différentes appellations pour désigner les Barbus ssp. A Bukavu, les Barbus ssp. Sont appelés "Katendere" ou "Kolongyo". A Kitutu, ils répondent au nom de "Kyumbi" ou "Shwe". A Kalemie, on les appelle "Kilangara”. A Kisangani, les gens les reconnaissent sous le nom de " Tematema. Par contre, à Bunyakiri, les gens reconnaissent les Labeobarbus altianalis sous le nom de "Nyalui".

\section{Position systématique et Description deLabeobarbusaltianalis}

Labeobarbus altianalis est une espèce de poissonappartenant au règne animalia, phylum chordata, sous-phylum craniata, super-classe de gnathostomata, grade de teleostomi, classe d'actinopterygii, sousclasse de neopterygii, division de Teleostei, sous-division d'Euteleostei, ordre de cypriniformes, super-famille de cyprinoïdae, famille de Cyprinidae, sous-famille de Cyprininae, genre Labeobarbus (Khady, 2015). Cette espèce a une coloration argentée, dorsalement gris-blanc et ventralement blanchâtre. La tête est brunâtre dorsalement et blanc-rose latéralement. On peut observer un reflet rosâtre sur le corps. Ses nageoires sont beiges à l'exception de la caudale qui est jaunâtre parfois avec une bande grise sur le lobe caudal inférieur (Figure 1).

\section{Traitement des échantillons}

Les poissons capturés à l'aide de ces filets sont triés par espèces. Pour éviter la poursuite de la digestion après la capture, du formol à 4\% était directement injectée dans la cavité viscérale de l'espèce concernée par l'étude de l'écologie alimentaire. Après cette injection le tout était conservé dans un bocal étiqueté contenant du formol à la même concentration pour une étude éventuelle au laboratoire. $\mathrm{Au}$ laboratoire, les diverses mensurations et observations ont été effectuées sur chaque poisson dont voici les détails :la longueur totale (L.T) : qui est la distance horizontale de l'extrémité de la nageoire caudale repliée c'est-à-dire la longueur maximale du poisson dans le sens horizontal; la longueur standard (L.S.) : la distance horizontale de l'extrémité antérieure du poisson à la base de la nageoire caudale, à l'endroit où le pédoncule caudal peut être plié aisément. Ces 2 longueurs ont été mesurées à l'aide d'un pied à coulisse précis jusqu'à $100^{\text {ème }} \mathrm{mm}$ et sont toutes exprimées en $\mathrm{mm}$. Le sexe $(\mathrm{S})$ : était déterminé après dissection et examen des gonades en se référant à l'échelle conventionnelle du degré de maturité inspirée de la mission de DE KIMPE (1946) et largement modifiée par Mutambue (1996) (Tableau 1). 


\section{Analyse des contenus digestifs}

L'analyse des contenus stomacaux des poissons constitue l'une des possibilités d'approcher la connaissance sur la présence, l'abondance et la disponibilité du potentiel alimentaire dans le milieu aquatique. Ces données apportent des connaissances sur les habitudes alimentaires du prédateur (Hartley 1948). Chez les poissons, l'étude du régime alimentaire se base souvent sur l'analyse de contenus stomacaux réalisée selon différentes méthodes (Lauzanne, 1975). Pour déterminer le régime et l'activité alimentaire de Labeobarbus altianalis, l'usage de l'indice alimentaire combinant l'indice volumétrique et indice d'occurence tels que utilisés par plusieurs auteurs (Lauzanne, 1976; Hyslop, 1980 ; Ulyel, 1991 ; Kraiem, 1980 ; Ponton et strottek, 1988; Benabid, 1990; ReyesMarchant et al., 1992) a été réalisé.

\section{La méthode d'occurrence ou de fréquence}

Elle consiste à compter le nombre d'estomacs où plusieurs individus d'unecertaine catégorie d'aliments sont représentés. Ce nombre est exprimé enpourcentage du nombre total d'estomacs non vides analysés (Plisnier et al 1988). On définit ainsi l'indice d'occurrence (Io) par :

\section{$\mathrm{Io}=\mathrm{Na} / \mathrm{Nt} X \mathbf{1 0 0}$.}

Où, $\mathrm{Na}$ : nombre d'estomacs où une catégorie d'aliments est présentée

$\mathrm{Nt}$ : nombre total d'estomac non vides analysés.

\section{Méthode volumétrique}

Cette méthode consiste à exprimer le volume d'une catégorie de proies pour l'ensemble de l'échantillon par rapport au volume total de l'ensemble de proies. Ce volume est mesuré en plaçant les catégories alimentaires dans un récipient gradué contenant de l'eau. Le déplacement d'eau permet l'estimation du volume des catégories alimentaires plongées dans le récipient (Hyslop, 1980).

L'indice volumétrique (Iv) se calcule selon la formule : $\mathbf{I v}=(\mathbf{V X} / \mathbf{V t}) \times \mathbf{1 0 0}$

Où : VX désigne le volume individuel de chaque catégorie de proies et

$\mathrm{Vt}$, le volume de toutes les catégories de proies.

\section{Indice alimentaire}

L'indice alimentaire (IA) permet de déterminer la nature réelle du régime alimentaire du poisson donné. Il tient compte non seulement des quantités observées de chaque catégorie alimentaire (Iv) ou (Iab) mais aussi de leur fréquence totale d'observation (Io). Cet indice combine l'indice volumétrique (Iv) ou d'abondance (Iab). Cet indice se calcule comme suit : Ia $=(\mathbf{I v} \times$ Io) $/ \mathbf{1 0 0}$ Où, Ia est l'indice alimentaire, Iv est l'indice volumétrique et Io est l'indice d'occurrence.

\section{Détermination de l'état de réplétion}

Pour déterminer l'état de remplissage d'estomac des Labeobarbus altianalis, nous nous sommes inspirés de la technique d'analyse de remplissage d'estomacs proposée par Ulyel1991. Cette technique consiste à attribuer aux estomacs certaines échelles des points en fonction de leur état de remplissage. Ces échelles des points sont réparties proportionnellement aux états de remplissage attribués visuellement et de façon subjective par l'analyste. Les échelles des points ayant été prises en compte sont les suivantes :

- Etat I : Plein soit 100 points

- Etat II : $3 / 4$ Plein soit 75 points

- Etat III : $1 / 2$ Plein soit 50 points

- Etat IV : 1/4 Plein soit 25 points et

- Etat V : Vide soit 0 point.

Cette détermination de remplissage de l'estomac, nous a permis d'avoir une idée sur le nombre d'estomacs vides et non vides des Labeobarbus altianalis capturés ; ce qui nous 
a permis de calculer les indices de réplétion et ceux de vacuité.

L'indice de réplétion (IR en \%) est le rapport entre le nombre d'estomacs non vides et le nombre d'estomacs analysés ou examinés multiplié par 100 (Ulyel, 1991). IR (\%) = $(\mathrm{Ep} / \mathrm{Et}) \times 100$, avec IR : indice de réplétion, Ep : nombre d'estomacs non vides,

Et : estomacs analysés ou examinés.

L'indice de vacuité (IV) est le rapport entre le nombre d'estomacs vides et le nombre d'estomacs analysés multiplié par 100 (Ulyel, 1991).

$\mathrm{IV}=(\mathrm{Ev} / \mathrm{Et}) \times 100$

où Ev est le nombre d'estomacs vides,

Et est le nombre d'estomacs examinés,

Iv est l'indice de vacuité.

\section{Identification des proies}

Après dissection, tout le tube digestif a été prélevé de l'œsophage jusqu'à l'anus. Leurs contenus étaient dilués dans $10 \mathrm{ml}$ d'eau et chaque fois $0,1 \mathrm{ml}$ d'eau était prélevé et placé sur la lame puis couvert de la lamelle, et cela après avoir homogénéisé l'échantillon. Les observations étaient réalisées grâce à un microscope de marque Olympus $\mathrm{CH}-30$ et Fr200MK2 de grossissement $10 \times 10$ et $40 \times 10$. Chaque fois que l'état des proies ingérées nous l'a permis, l'identification a été réalisée au niveau générique ou spécifique à partir des ouvrages de détermination décrits par plusieurs auteurs (Mpawenayo, 1996 ; Fernando, 2002 ; Ramade 2003, 2005 ; Isumbisho et al., 2006). Afin d'éviter toute erreur, les proies en état de décomposition très avancée sont classées dans la rubrique «Indéterminés». Le nombre des proies qui ne sont que partiellement avalées ou qui ont tendance à se fragmenter pendant la digestion, a été déduit du comptage des parties du corps facilement identifiables telles que les yeux de crustacés, les disques d'astérides, les becs de céphalopodes.

\section{Caractérisation du régime alimentaire}

Pour caractériser le régime alimentaire des Labeaobarbus altianalis capturés, nous avons calculé trois indices, notamment l'indice d'occurrence et l'indice volumétrique. Ces deux derniers indices nous ont conduit à calculer l'indice alimentaire en utilisant la formule suivante : Ia $=(\mathrm{Io} \times \mathrm{Iv}) / 100$. Ainsi, Lauzanne classa les proies en quatre catégories :

$\checkmark$ Pour Ia $<$ à $10 \%$ : proies d'importance secondaire,

$\checkmark$ Pour Ia compris entre 10 et $24 \%$ : proies importantes,

$\checkmark$ Pour Ia compris entre 25 et $50 \%$ : proies essentielles,

$\checkmark$ Pour Ia $>$ à $50 \%$ : proies largement dominantes

\section{Rythme d'activité alimentaire}

Pour déterminer le moment d'alimentation, nous avons calculé pour chaque heure de capture, la proportion (en \%) du nombre d'estomacs non vides par rapport au nombre total d'estomacs analysés. La variation de ces pourcentages au cours du cycle de 12 heures renseigne sur les périodes ou moment d'alimentation des poissons. Le nombre des poissons capturés par tranche horaire a été noté pour permettre d'examiner si les captures des poissons reflètent bien leur activité alimentaire puisque ceux-ci pourraient se faire attraper dans les filets lors de la recherche de la nourriture.

\section{Analyse statistique}

Les données pour cette étude ont été analysées par le logiciel SPSS version 20 et le classeur EXCEL. 


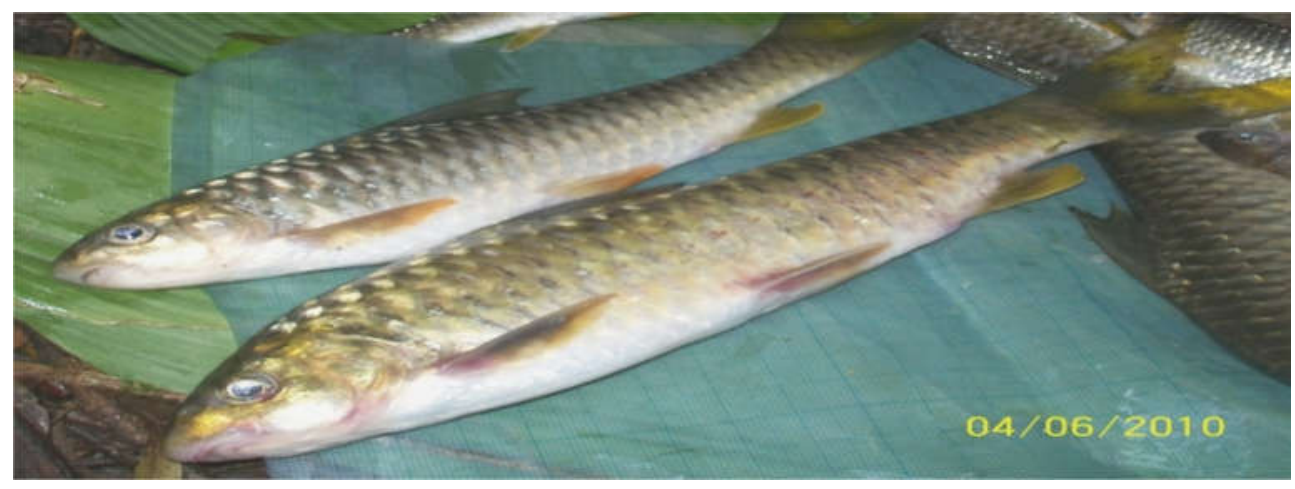

Figure 1 : Labeobarbus altianalis de l'affluents de la rivière Luhoho

Tableau 1 : Caractéristiques de divers stades de maturité sexuelle (Mutambue, 1996).

\begin{tabular}{|c|c|c|c|}
\hline STADES & & MALES & FEMELLES \\
\hline \multirow[t]{2}{*}{ I } & - & $\begin{array}{l}\text { - Gonades de petites dimensions } \\
\text { accolées à la colonne vertébrale } \\
\text { Sexe non reconnaissable même au } \\
\text { binoculaire }\end{array}$ & $\begin{array}{l}\text { - Femelle en repos sexuel: ovaires peu } \\
\text { développés en forme de bandelettes. } \\
\text { Ovocytes visibles et enveloppés par un } \\
\text { tissu à apparence spongieuse. Lamelles } \\
\text { ovariennes présentes et vascularisation } \\
\text { externe non marquée. }\end{array}$ \\
\hline & & & $\begin{array}{l}\text { Sexe non reconnaissable même au } \\
\text { binoculaire }\end{array}$ \\
\hline $1 / 4$ & & $\begin{array}{ll}\text { - } & \text { Gonades transparentes à } \\
\text { l'observation }\end{array}$ & $\begin{array}{l}\text { Gonades transparentes à l'observation et } \\
\text { ovocytes discernables au binoculaire }\end{array}$ \\
\hline \multirow[t]{2}{*}{$2 / 4$} & & $\begin{array}{l}\text { - Testicules rose- rougeâtres ou } \\
\text { rose- blanchâtres }\end{array}$ & $\begin{array}{l}\text { - Ovaires rosâtres ou rose- claires ou } \\
\text { rougeâtres d'aspect granuleux }\end{array}$ \\
\hline & & & $\begin{array}{l}\text { - Ovocytes bien visibles mais non } \\
\text { dissociables }\end{array}$ \\
\hline \multirow[t]{2}{*}{$3 / 4$} & & $\begin{array}{l}\text { - Testicules blanchâtres ou rose- } \\
\text { blanchâtres bien développés avec } \\
\text { les points de laitance }\end{array}$ & $\begin{array}{l}\text { - Ovaires jaunâtres ou rose- jaunes ou jaune- } \\
\text { blanchâtres }\end{array}$ \\
\hline & & & - $\quad$ Ovules bien visibles et dissociables \\
\hline \multirow[t]{2}{*}{$4 / 4$} & & $\begin{array}{l}\text { - Testicules blanchâtres, laitance } \\
\text { expulsable par pression du doigt }\end{array}$ & $\begin{array}{l}\text { - Ovaires jaunes, jaunes- orange ou jaune- } \\
\text { citron }\end{array}$ \\
\hline & & - $\quad$ Spermiducte gorgé de laitance & - $\quad$ Présence d'ovules dans l'oviducte \\
\hline $5 / 4$ & & $\begin{array}{l}\text { - Testicules rose- pâle flasques et } \\
\text { vidés de spermes }\end{array}$ & $\begin{array}{l}\text { - Ovaires roses ou rouges- brique, vides, } \\
\text { flasques, quelques granulations blanchâtres } \\
\text { petites }\end{array}$ \\
\hline
\end{tabular}




\section{RESULTATS}

\section{Résultats de pêche (capture totale)}

Abondance des taxa et évolution des captures dans les quatre sites choisis.

En ce qui concerne l'abondance de différents taxa, le Tableau 2 montre que Labeobarbus altianalis (anciennement appélé Barbus altianalis Boulenger) était plus abondant $(63,7 \%)$ dans tous les sites suivi de Clarias theodorae qui occupe la deuxième position du point de vue abondance (12,5\%) sur l'ensemble des sites. Chiloglanis lukugae quant à lui représente $11,9 \%$ de la capture totale et occupe ainsi la troisième position. Les autres taxa étaient faiblement représentés au cours de l'échantillonnage pendant un cycle de 12 heures. Il relève en outre que sur les quatre sites considérés, le site de Tchinganda vient en tête $42,3 \%$ d'espèces capturées suivi du site Eke puis Tchoko respectivement avec $22,5 \%$ et $20,3 \%$ d'espèces capturées. Le site de Nyamunene occupe la dernière position en termes d'abondance d'espèce. Parlant de l'évolution de pêche, la Figure 2 montre que les captures étaient plus bonnes le matin et au milieu de la journée (entre $6 \mathrm{~h}-12 \mathrm{~h}^{00}$ ) à Tchinganda et Eke tandis que dans les sites de Tchoko et Nyamunene, elles se sont révélées nulles pendant ce temps mais plus élevées le soir (entre $\left.14 \mathrm{~h}-18 \mathrm{~h}^{00}\right)$. Les pics de captures montrent une activité intense des taxa en général. Le tableau 2 montre que Labeobarbus altianalis ont été capturé à toutes les tranches horaires avec un pic au lever du soleil $\left(6 \mathrm{~h}^{00}\right.$ $9 \mathrm{~h}^{00}$ ) dans Tchinganda et Eke. La situation s'est présentée autrement à Tchoko et à Nyamunene où cette espèce a été capturée seulement le soir (Figure 2).

\section{Quelques données démographiques de}

\section{Labeobarbus altianalis}

\section{Sex-ratio et fréquence des tailles}

L'analyse de résultats obtenus montre que les mâles ont dominé l'échantillon avec un taux de représentation de plus de $60 \%$ dans trois sites et un sex-ratio de 2,$1 ; 2$ et 1,7 respectivement à Tchiganda, Tchoko et Eke. La situation s'est présentée autrement à Nyamunene où les femelles dominent les mâles. Le sex-ratio (rapport entre les mâles et les femelles) appliqué à cet échantillon donne 0,3 . (Tableau 4) Sur $100 \%$ de spécimen analysé $(\mathrm{n}=235), 70,2 \%$ de nos échantillons avaient la taille comprise entre 89,00-118,99 mm. $22,2 \%$ étaient composés des individus dont la taille varie entre 119,00-178,99 mm. Le reste de classes ont été les moins représentées dans la capture et se partagent le $7,6 \%$ restant (Figure 3).

Fréquence des stades de maturité des gonades

Sur 235 spécimen de Labeobarbus altianalis analysés, les individus à stade $2 / 4$ ont dominé nos échantillons $(63,0 \%)$, suivis de ceux du stade $3 / 4$ avec $17 \%$, ceux en période reproductive (stade $4 / 4$ et 5/4) étaient les moins représentés avec respectivement $13,6 \%$ et $6,4 \%$ tandis que ceux au stade 1 et $1 / 4$ n’ont pas été observés (Figure 4).

\section{Ecologie alimentaire de Labeobarbus altianalis \\ Composition quantitative et qualitative du régime alimentaire de Labeobarbus altianalis dans les quatre sites d'échantillonnages}

La composition du régime alimentaire de Labeobarbus altianalis était dominée par le phytoplancton des groupes de Bacillariophytes (représentée par les genres Navicula, Nitzchia, Eunotia, Fragilaria, Frustilia, Gomphonema et Tabellaria). Cyanophytes (du genre Anabaena, Anabaenopsis, Aphanocapsa, Bacillaria, Chloococus, Microcystis et Oscillatoria). Le groupe de Chlorophycées était représenté par les genres Ankistrodesmus, Characium, Cosmarium, Coelastrum, Cyclidiopsis, Closterium, Monoraphidium, Merismopodia, Pediastrum, Pleurotaeniul, Tetra, Sporaraspora. Le groupe d'Euglenophytes était représenté quant à lui par deux genres, Euglena et Tachelomonas, des insectes, des 
écailles et débris végétaux, le phytoplancton. Les Cladocères, Rotifères et Copépodes constituaient le zooplancton tandis que les insectes étaient représentés par les larves des Chironomidés. On a aussi noté, dans les estomacs de spécimen de Labeobarbus altianalis, la présence des débris végétaux et des crevettes.

Sur le plan qualitatif, sur Ainsi dans tous les sites, Labeobarbus altianalis s'alimente plus du phytoplancton, des débris végétaux (Io $=78,8 \%$ ), des insectes (Io $=$ $55,5 \%$ ), du zooplancton (Io $=52,2 \%$ ), crevettes (Io $=24,4 \%$ ) et écailles (Io $=$ 17,7\%).S'agissant du volume de chaque catégorie de proies (indice volumétrique) Labeobarbus altianalis a consommé quantitativement $\mathrm{du}$ phytoplancton (Iv = $99,9 \%$ ) et des débris végétaux ( $\mathrm{Iv}=0,8 \%$ ) et en faible quantité les autres groupes des proies (Tableau 5). En combinant les aspects qualitatifs et quantitatifs, nous remarquons par le tableau 5 que, pour Labeobarbus altianalis, le phytoplancton constitue la proie largement dominant dans tous les sites considérés ( $\mathrm{Ia}=$ 98,8 à Tchinganda, Ia $=88,7 \%$ à Tchoko, Ia $=$ $88,8 \%$ à Eke et Nyamunene, Ia $=99 \%$ ). Cependant, il complète sa ration alimentaire avec les débris végétaux, le zooplancton, les crevettes et les écailles, dont les indices alimentaires étaient inférieurs à $10 \%$. Ils constituent ainsi les proies secondaires pour cette espèce (Tableau 6).

\section{Variation du régime alimentaire de Labeobarbus altianalis selon la taille}

Les valeurs des indices alimentaires des différentes proies ingérées par Labeobarus altianalis dans toutes les tailles des juvéniles aux adultes montrent que les phytoplanctons sont classés en proie largement dominant. Les adultes complètent ce régime par les débris végétaux et le zooplancton. Ainsi Labeobarbus altianalis, poisson omnivore et d'habitude planctonophage, révèle au bassin de la rivière Luhoho une tendance phytophage. Ceci nous a poussé à étudier les variations de l'indice alimentaire en fonction de la taille des poissons, dans le but de mieux caractériser les tendances alimentaires des Labeobarbus altianalis au cours de leur vie dans ces cours d'eaux (Figure 5).

En effet, l'analyse de l'indice d'occurrence des types des aliments fait marquer que Labeobarbus altianalis des affluents de la rivière Luhoho se nourrit préférentiellement $\mathrm{du}$ phytoplancton $\mathrm{du}$ groupe de Bacillariophytes, Cyanophytes, Chlorophytes et Euglenophytes (Io $=100 \%$ ) à toutes les classes de taille et dans tous les sites. Ce poisson se nourrit également des débris végétaux et zooplancton dont les indices d'occurrence sont supérieurs à $50 \%$ à tous les âges. Signalons cependant que les insectes du groupe de Chironomidés ont les indices d'occurrence supérieurs à $50 \%$ pour les individus dont la taille varie entre 119,00 et $208,99 \mathrm{~mm}$. On constate également que Labeobarbus altianalis complète régulièrement sa ration alimentaire presque à tous les âges avec les écailles. Enfin, ce tableau montre également que Labeobarbus altianalis fait de sélections pour certaines nourritures selon l'âge. C'est ainsi qu'on ne trouve pas les crevettes dans les estomacs de ce poisson à l'âge compris entre 179,00 $268,99 \mathrm{~mm}$ et les insectes pour les individus à l'âge de 209,00 - 268,00 mm.

S'agissant du volume de chaque catégorie de proies, le Tableau 6 montre que Labeobarbus altianalis a consommé quantitativement du phytoplancton (Iv est plus de $80 \%$ ) à tous les âges suivi des débris végétaux (Iv est plus ou moins égal à $5 \%$ dans l'ensemble des sites considérés. Les autres catégories de proies étaient consommées en 
faible quantité. L'indice alimentaire déterminé à partir de la combinaison des indice d'occurrence et volumétrique, permet de dire queLabeobarbus altianalis des affluents de la rivière Luhoho, se nourrit préférentiellement $\mathrm{du}$ phytoplancton dont les indices d'alimentaires sont supérieurs à $50 \%$ à tous les âges (sauf à la classe d'âge compris entre 209,00 et $238,99 \mathrm{~mm}$, où il apparaît comme essentielle). Il complète sa ration alimentaire avec le zooplancton, les débris végétaux et les écailles dont les indices alimentaires (Ia) sont respectivement égaux à $30 \%, 12 \%$ et $15,6 \%$ pour les individus de taille entre 209,00$238,99 \mathrm{~mm}$. Les proies comme les crevettes et les insectes ont été observés rarement dans les estomacs de ce poisson. Constatons également que ces proies n'ont pas été consommées par les Labeobarbus altianalis des affluents de la rivière Luhoho (Tableau 7). Eu égard à ce qui précède, on voit que les Labeobarbus altianalis sont des poissons phytoplanctonophages complétant leur ration alimentaire par les débris végétaux.

\section{Rythme d'activité d'alimentation de Labeobarbus altianalis

Indices de réplétion et moment
d'alimentation

L'analyse des indices de réplétion stomacale fonction du moment d'échantillonnage dans les quatre sites d'échantillonnages. Au cours de cette étude, 235 estomacs de Labeobarbus altianalis dont 220 non vides ont été analysés sur l'ensemble des sites considérés et relève les faits ciaprès: A Tchinganda, l'indice de réplétion stomacale a varié en fonction de l'évolution de la capture au moment de l'échantillonnage. C'est ainsi qu'on remarque les fortes réplétions à chaque moment de capture (pêche) quel que soit le nombre d'individus capturés. Tandis qu'à Tchoko et à Nyamunene les faibles captures (réplétion) se sont constituées au lever du soleil et au milieu de la journée. On notera dans ces deux sites (Tchoko et Nyamunene), les fortes captures seulement le soir. A Eke par contre, les fortes réplétions se sont observées le matin et au milieu de la journée. Dans l'ensemble de quatre sites, l'indice de réplétion est plus élevé le matin suivi de celui du soir. Une explication serait que, le matin l'espèce commence à se nourrir et atteint le pic de son activité alimentaire à $18 \mathrm{~h}$. Au milieu de la journée, pendant qu'il fait clair, l'espèce entre au repos ainsi on remarque une diminution $\mathrm{du}$ volume des estomacs. Cette activité de ce poisson est liée à la recherche de la nourriture (Figure 6). Il existe un lien étroit entre l'activité locomotrice de ce poisson et son alimentation.

\section{Evolution des proies consommées par Labeobarbus altianalis}

D’une manière générale, le phytoplancton suivi de Débris végétaux ont constitué la majorité des proies consommées par Labeobarbus altianalis dans les quatre sites d'échantillonnage. Quant au site de Tchinganda et EKE, le phytoplancton est en première position tandis qu'on remarque pendant la journée et au coucher du soleil que les débris végétaux constituent le second groupe de proie en termes d'abondance. Le phénomène s'observe autrement dans le site de Tchoko et Nyamunene où une grande consommation des débris végétaux et du zooplancton occupe la deuxième place dans les deux autres sites, le soir. (Figure 7). 
Tableau 2 : Relation entre les sites de pêche et abondance de taxa capturé.

\begin{tabular}{lllllll}
\hline \multicolumn{1}{c}{ Espèce } & Tchinganda & Tchoko & Eke & Nyamunene & TOTAL & \% \\
\hline Labeobarbus altianalis & 117 & 40 & 49 & 29 & 235 & 63,7 \\
Schiloglanis lukugae & 15 & 12 & 10 & 7 & 44 & 11,9 \\
Oreochromis niloticus & 6 & 4 & 9 & 2 & 21 & 5,7 \\
Clarias theodorae & 13 & 13 & 12 & 8 & 46 & 12,5 \\
Bryconacthiops boulangeri & 3 & 4 & 2 & 6 & 15 & 4,1 \\
Opsaridium spp & 2 & 2 & 1 & 3 & 8 & 2,2 \\
\hline TOTAL & $\mathbf{1 5 6}$ & $\mathbf{7 5}$ & $\mathbf{8 3}$ & $\mathbf{5 5}$ & $\mathbf{3 6 9}$ & $\mathbf{1 0 0 , 0 0}$ \\
\hline Pourcentage & 42,3 & 20,3 & 22,5 & 14,9 & $\mathbf{1 0 0}$ & \\
\hline
\end{tabular}

Tableau 3 : Abondance de Labeobarbus altianalis capturés entre 6h-18heures dans les quatre sites d'échantillons ( $\mathrm{n}$ : effectif, \% : pourcentage).

\begin{tabular}{lllll}
\hline Site & $\mathbf{6 h} \mathbf{- 9 h}$ & $\mathbf{1 0 h} \mathbf{- 1 2 h}$ & $\mathbf{1 4 h} \mathbf{- 1 8 h}$ & TOTAL \\
\hline Tchinganda & 54 & 35 & 28 & $\mathbf{1 1 7}$ \\
Tchoko & 8 & 10 & 22 & $\mathbf{4 0}$ \\
Eke & 16 & 26 & 7 & $\mathbf{4 9}$ \\
Nyamunene & 11 & 10 & 8 & $\mathbf{2 9}$ \\
\hline TOTAL & $\mathbf{8 9}$ & $\mathbf{8 1}$ & $\mathbf{6 5}$ & $\mathbf{2 3 5}$ \\
\hline
\end{tabular}

Tableau 4 : Proportion (en \%) des mâles et de femelles de Labeobarbus altianalis par site ( $\mathrm{n}=$ effectif de l'échantillon, s-r = sex-ratio et $\mathrm{N}=$ effectif total).

\begin{tabular}{lllll}
\hline \multirow{2}{*}{ Sexe } & Sites & & & \\
\cline { 2 - 5 } & Tchinganda & Tchoko & Eke & Nyamunene \\
\hline Mâle & 68,1 & 66,6 & 62,5 & 25 \\
Femelle & 31,9 & 33,4 & 37,5 & 75 \\
\hline Total & 100 & 100 & 100 & 100 \\
\hline $\mathrm{n}$ & 117 & 40 & 49 & 29 \\
\hline s-r & 2,1 & 2 & 1,7 & 0,3 \\
\hline
\end{tabular}


Tableau 5: composition générale du régime alimentaire de Barbus altianalis (Io: indice d'occurrence et Iv est l'indice volumétrique).

\begin{tabular}{|c|c|c|c|c|c|c|c|c|c|c|c|c|}
\hline \multirow{2}{*}{$\begin{array}{l}\text { Type des } \\
\text { proies }\end{array}$} & \multicolumn{2}{|c|}{$\begin{array}{l}\text { 89,00-118,99 } \\
\text { mm }\end{array}$} & \multicolumn{2}{|c|}{$\begin{array}{l}119,00- \\
148,99 \mathrm{~mm}\end{array}$} & \multicolumn{2}{|c|}{$\begin{array}{l}149,00- \\
178,99 \mathrm{~mm}\end{array}$} & $\begin{array}{l}179,00- \\
208,99 \\
\text { mm }\end{array}$ & $\begin{array}{l}209,00- \\
238,99 \\
\mathrm{~mm} \\
\end{array}$ & \multicolumn{2}{|c|}{$\begin{array}{l}239,00- \\
268,00 \mathrm{~mm}\end{array}$} & \multirow[b]{2}{*}{$\operatorname{Io}(\%)$} & \multirow[b]{2}{*}{ Iv(\%) } \\
\hline & $\operatorname{Io}(\%)$ & $\operatorname{Iv}(\%)$ & Io(\%) & $\operatorname{Iv}(\%)$ & $\operatorname{Io}(\%)$ & $\operatorname{Iv}(\%)$ & Io(\%) & $\operatorname{Iv}(\%)$ & Io(\%) & $\operatorname{Iv}(\%)$ & & \\
\hline Zooplancton & 54,6 & 3,2 & 47,3 & 2,9 & 33,3 & 0,6 & 60 & 1,9 & 100 & 30,8 & 0 & 0 \\
\hline Phytoplancton & 100 & 84,1 & 100 & 86,2 & 100 & 89,4 & 100 & 86,9 & 100 & 41,6 & 100 & 83,5 \\
\hline Crevettes & 23,7 & 1,9 & 15,7 & 0,9 & 22,2 & 0,5 & 0 & 0 & 0 & 0 & 0 & 0 \\
\hline Insectes & 45,3 & 1,8 & 52,6 & 1,5 & 55,5 & 0,8 & 80 & 0,5 & 0 & 0 & 0 & 0 \\
\hline $\begin{array}{l}\text { Débris } \\
\text { végétaux }\end{array}$ & 74,22 & 8,9 & 63,1 & 6,6 & 77,7 & 6,1 & 80 & 9,3 & 100 & 12 & 100 & 16,5 \\
\hline Ecaille & 13,4 & 0,1 & 5,2 & 1,9 & 11,1 & 2,6 & 40 & 1,4 & 10 & 15,6 & 0 & 0 \\
\hline
\end{tabular}

Tableau 7 : Régime alimentaire général de Labeobarbus altianalis.

\begin{tabular}{lllll} 
Types de Proies & \multicolumn{1}{l}{ Sites } & & & \\
\cline { 2 - 4 } & Tchinganda & Tchoko & Eke & Nyamunene \\
\hline Zooplancton & 0,2 & 1,6 & 3,5 & 0,5 \\
Phytoplancton & 98,8 & 88,7 & 88,8 & 99,0 \\
Crevettes & 0,1 & 0,5 & 0,0 & 0,0 \\
Insectes & 0,2 & 0,6 & 1,1 & 0,0 \\
Débris végétaux & 0,6 & 3,9 & 0,7 & 0,0 \\
Ecaille & 0,1 & 0,0 & 0,0 & 0,0 \\
\hline
\end{tabular}


Tableau 6: Composition du régime alimentaire de Labeobarbus altianalis en fonction de la taille.

\begin{tabular}{|c|c|c|c|c|c|c|c|c|}
\hline \multirow{3}{*}{ Types de Proies } & \multicolumn{8}{|l|}{ Sites } \\
\hline & \multicolumn{2}{|c|}{ Tchinganda } & \multicolumn{2}{|l|}{ Tchoko } & \multicolumn{2}{|l|}{ Eke } & \multicolumn{2}{|c|}{ Nyamunene } \\
\hline & Io (\%) & Iv (\%) & Io $(\%)$ & Iv (\%) & Io (\%) & Iv (\%) & Io (\%) & Iv (\%) \\
\hline Zooplancton & 52,2 & 0 & 66,6 & 2,4 & 62,5 & 5,6 & 50 & 0,9 \\
\hline Phytoplancton & 100 & 99 & 100 & 88,7 & 100 & 88,8 & 100 & 99,0 \\
\hline Crevettes & 24,4 & 0 & 26,6 & 2,0 & 1,1 & 1,4 & 0 & 0,0 \\
\hline Insectes & 55,5 & 0 & 60 & 1,0 & 75 & 1,5 & 25 & 0,1 \\
\hline Débris végétaux & 78,8 & 1 & 66,6 & 5,9 & 25 & 2,7 & 0 & 0,0 \\
\hline Ecaille & 17,7 & 0 & 0 & 0,0 & 0 & 0 & 0 & 0 \\
\hline
\end{tabular}

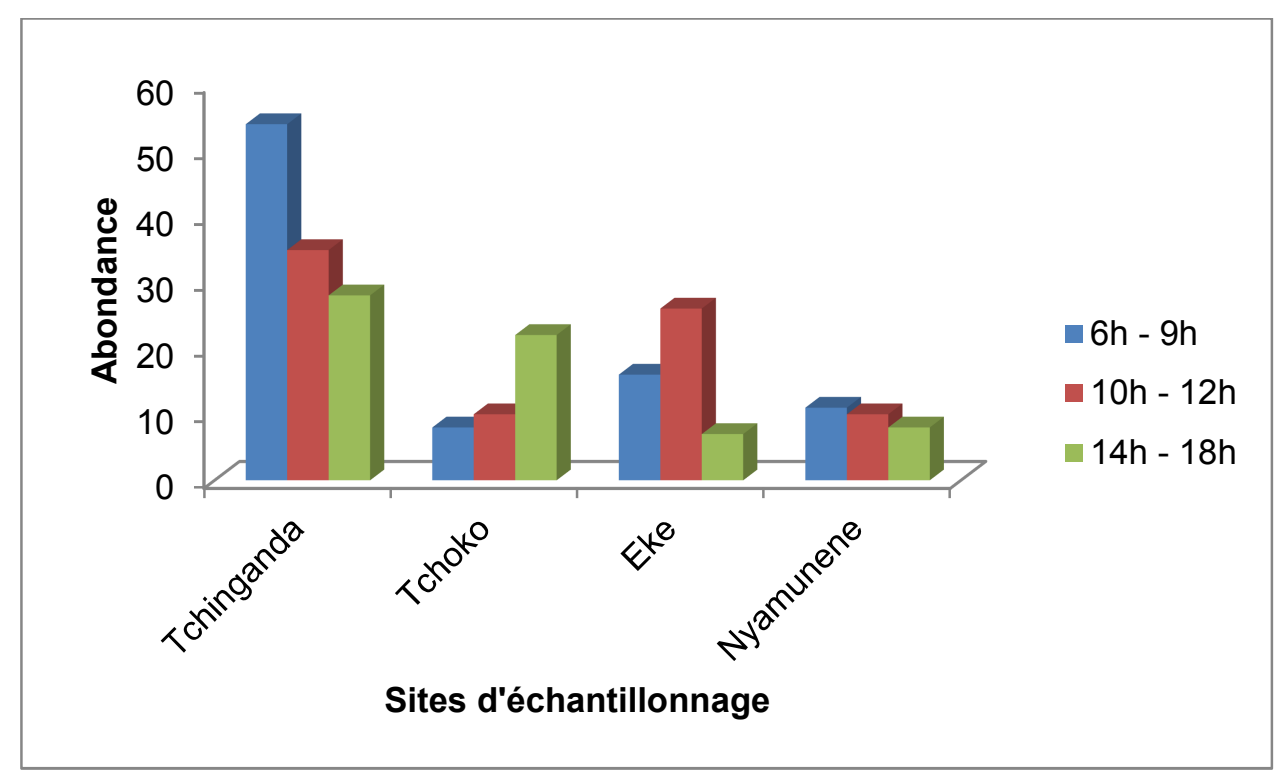

Figure 2 : Variation des captures par tranche horaire selon les sites. 


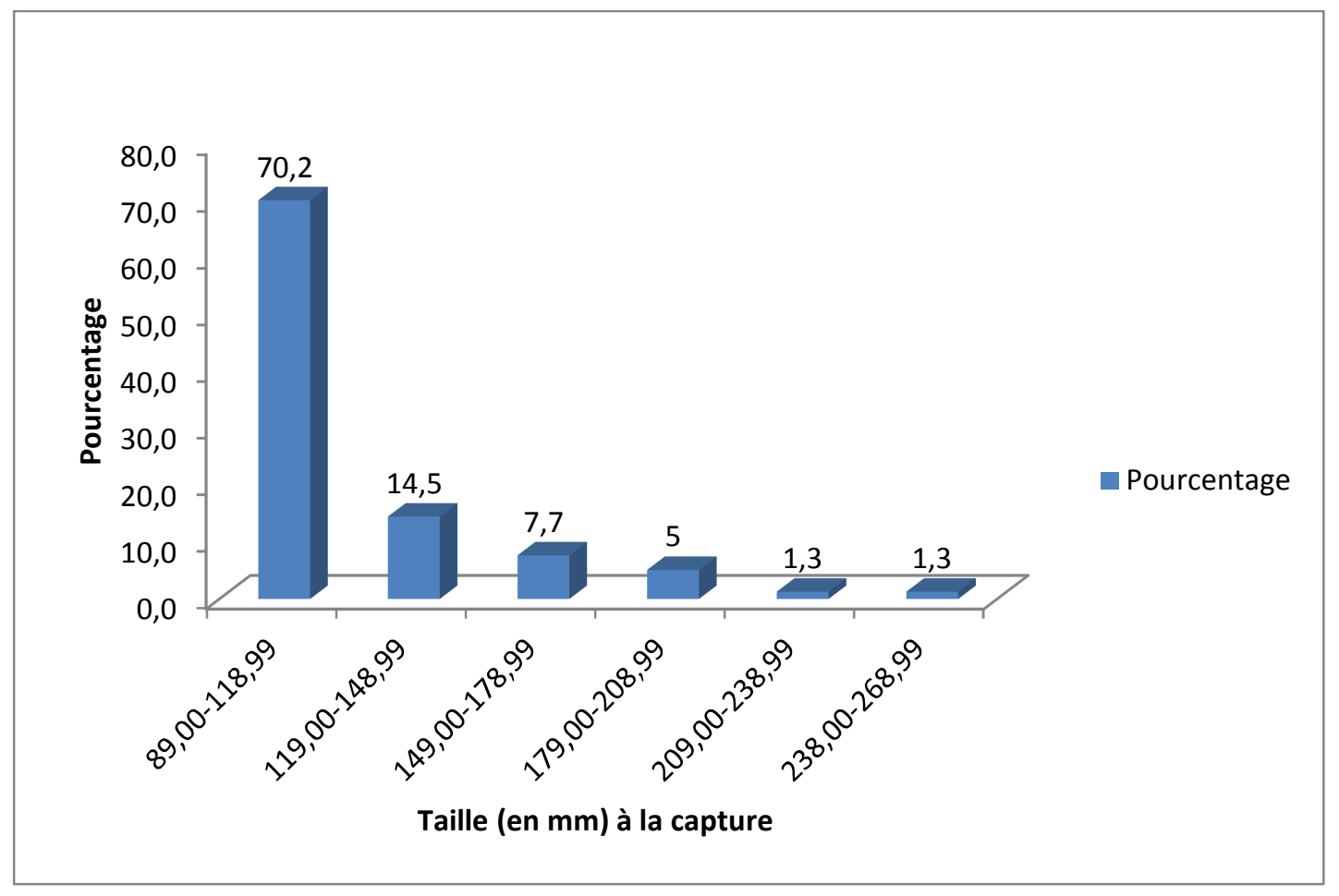

Figure 3 : Fréquence des Labeobarbus altianalis selon la taille.

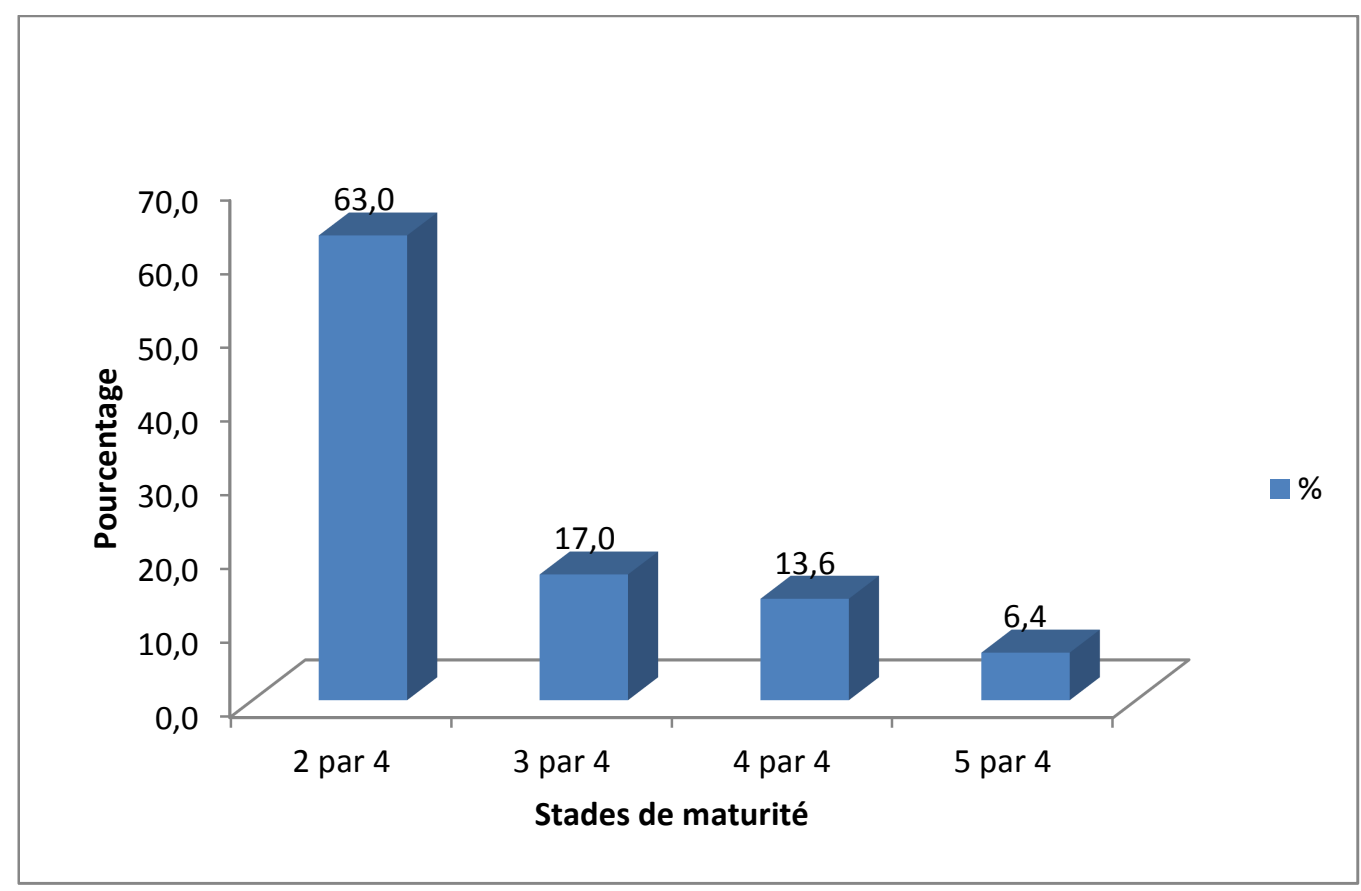

Figure 4: Fréquence des stades de maturité des gonades de Labeobarbus altianalis. 


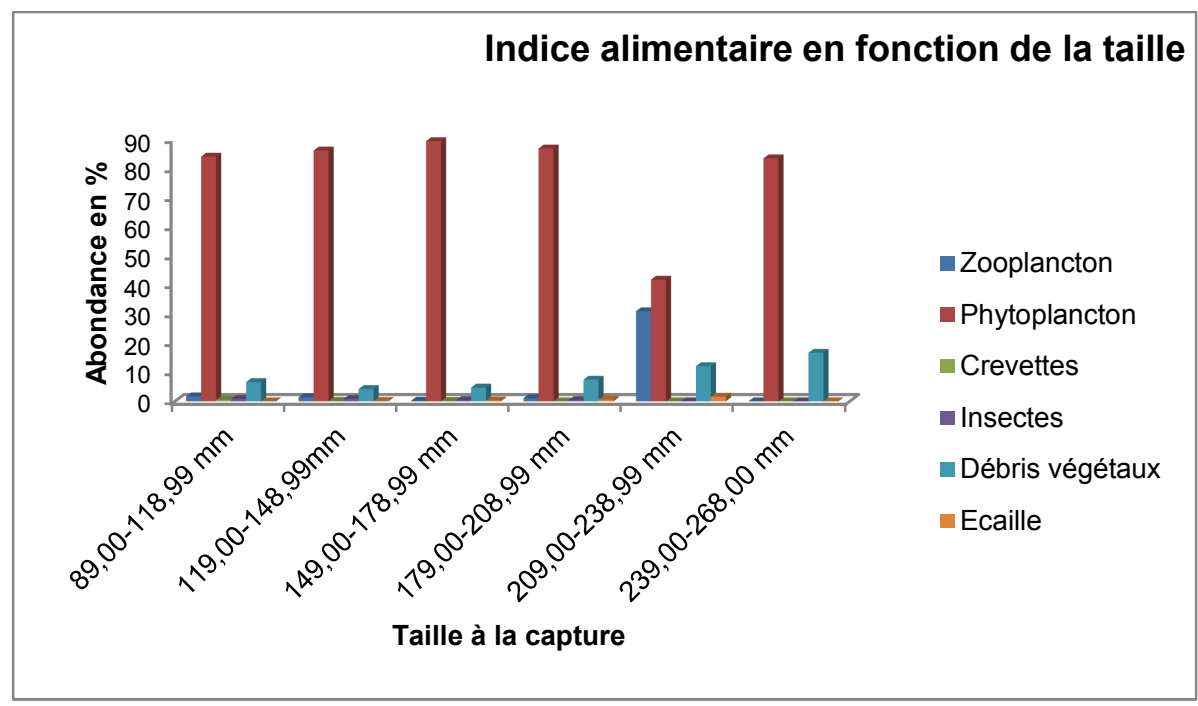

Figure 5 ; Intensité de prédation de Labeobarbus altianalis en fonction de la taille.
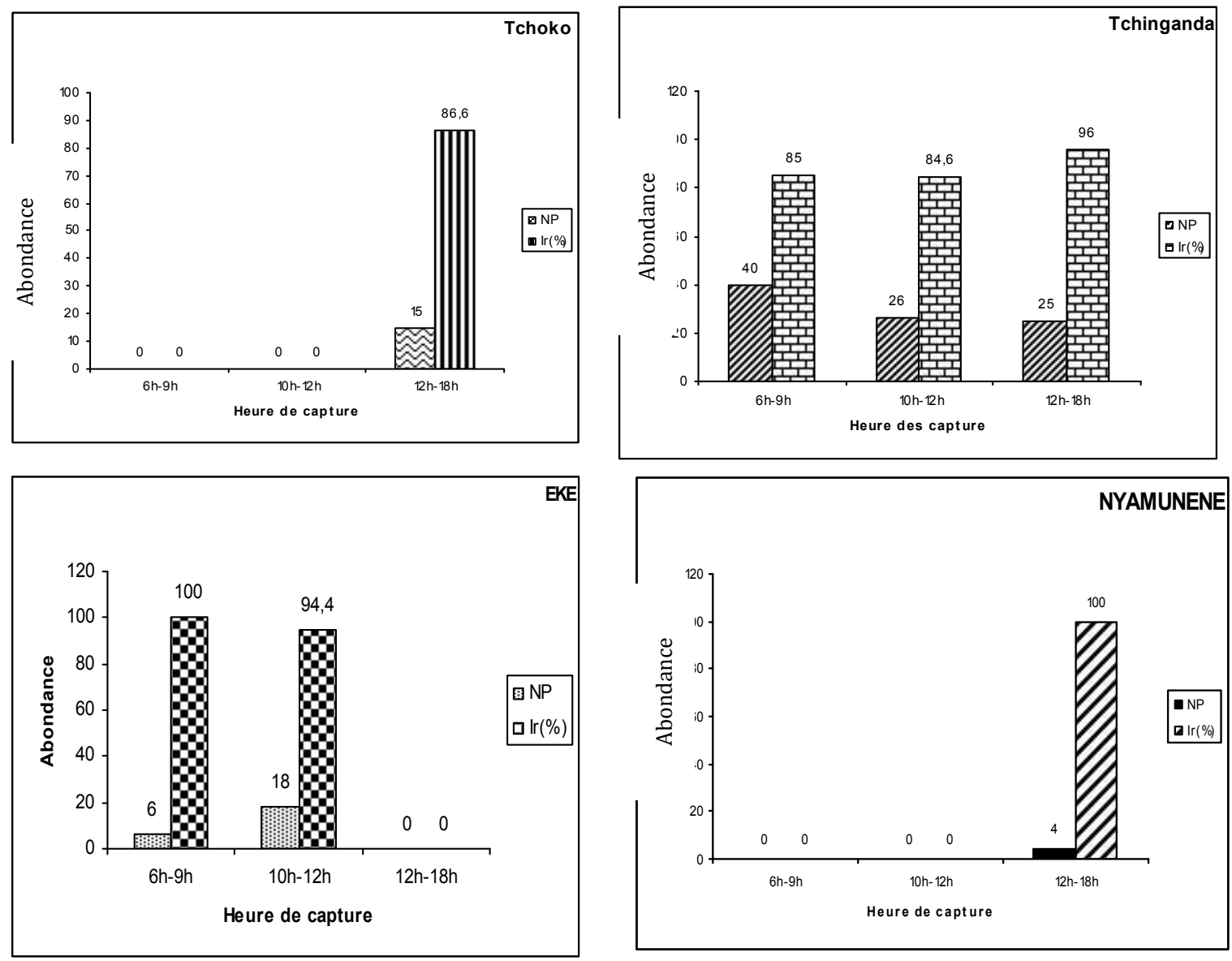

Figure 6 : Evolution de capture et les indices de réplétion pour Labeobarbus altianalis par site. 

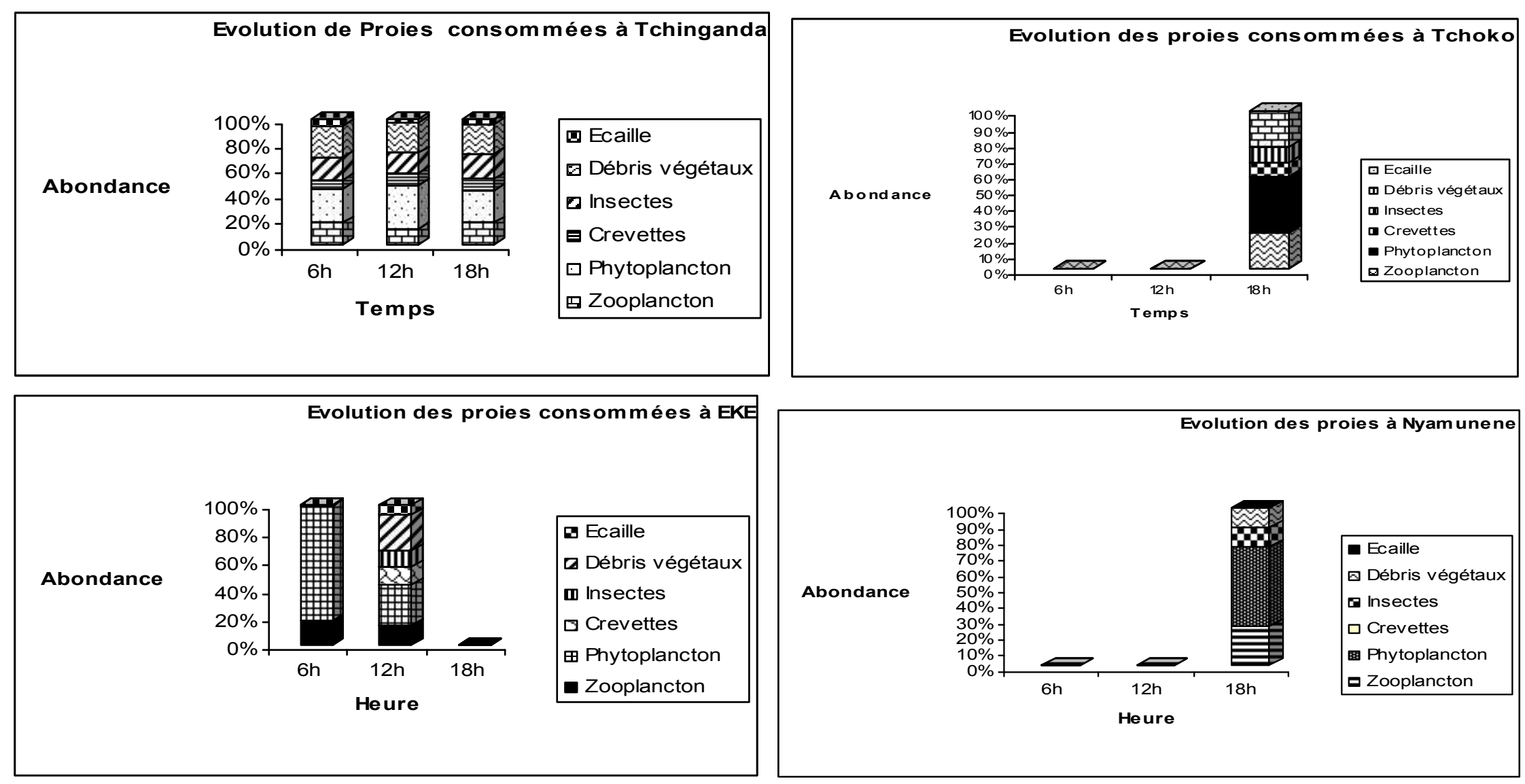

Figure 7 : Comparaison des types d'alimentations consommés par Labeobarbus altianalis dans différant sites. 


\section{DISCUSSION}

La connaissance de la richesse de tout écosystème aquatique constitue l'étape préliminaire d'une gestion scientifique et cette connaissance conduit au développement de toutes les activités qui doivent s'y dérouler car tout milieu est en évolution dans le temps et cette évolution conduit à des changements physiques, chimiques et biologiques du milieu.

Au cours de cette étude, il a été trouvé, lors du sexage, les individus avec trois stades de maturité, le stade $2 / 4,3 / 4$ et $4 / 4$ dans tous les sites avec la dominance numérique des individus au stade $2 / 4$. On a trouvé les œufs dans les gonades des individus de $94,67 \mathrm{~mm}$ de longueur totale. Au regard de ce qui précède, nous sommes limité de dire avec exactitude, la période de reproduction de cette espèce étant donné que l'échantillonnage n'a pas été répétitif dans tous les sites durant toute une année. Néanmoins, tout ce qu'on peut dire à ce sujet, c'est que les échantillons pour cette étude étaient dominés par les subadultes (2/4) que les adultes (stade $3 / 4$ et $4 / 4$ ). Pour ce qui est de la taille des individus des Labeobarbus altianalis capturés, le plus petit spécimen de nos échantillons avait une taille de 89,07 mm de longueur totale et le plus grand individu avait $246,00 \mathrm{~mm}$ de longueur totale. Cette taille est largement inférieure à celle trouvée au lac Kivu pour la même espèce (Waasalo 2003). Cet auteur a trouvé que cette espèce mesure $560 \mathrm{~mm}$ de longueur totale; les milieux différents (lotique et lentique) dans lesquels vit cette espèce seraient à la base de cette différence en ce sens que la composition change d'un milieu à l'autre.

Les captures numériques de Labeobarbus altianalis se sont révélées plus fructueuses à Tchinganda qu'aux autres sites. Ceci pourrait s'expliquer par l'effort de pêche consenti dans ce site que dans d'autres. Le rythme d'activité locomotrice de cette espèce dans l'ensemble de quatre sites indique que les pics des captures de Labeobarbus altianalis observés à Tchinganda montrent qu'ils se déplacent le matin, midi et soir (6h18h). A Tchoko et à Nyamunene, c'est le soir (18heures) que cette espèce se déplace intensément. Signalons toutefois qu'à Eke, c'est entre 6heures et 12 heures qu'ils étaient plus capturés. Les Labeobarbus altianalis se nourrissent à Tchinganda et à Eke le jour (6h12heures) comme le soir (18heures). Ce rythme d'activité alimentaire est qualifié de bimodal. Ses heures de nourrissage correspondraient aux heures de disponibilités des proies préférées par Labeobarbus altianalis. Pour soutenir l'idée ci-haut évoquée, plusieurs auteurs suggèrent que la plupart des activités alimentaires impliqueraient des mouvements de repos vers des zones d'alimentation suivant une périodicité pouvant être liée au rythme d'activités des proies (Cherghou, 2002; Mamadou et Ghorbel, 2010).

Le rythme alimentaire peut être annuel (saisonnier) ou journalier, diurne et/ou nocturne, crépusculaire, unimodal, rythmique ou arythmique; ce dernier cas étant plus rare en milieu naturel (Cikwanine, 2008). Il se caractérise par sa périodicité, son intensité et par son mode. L'analyse des indices alimentaires de Labeobarbus altianalis des affluents de la rivière Luhoho (Tchinganda, Tchoko, Eke et Nyamunene) montre que cette espèce se nourrit essentiellement du phytoplancton. L'on peut dire que cette espèce est phytoplanctonophage bien qu'elle s'alimente aussi des débris végétaux, de zooplancton, des crevettes et des insectes à presque tous les âges. En effet, Labeobarbus altianalis est caractérisée par des nombreuses branchiospines sur la première partie de l'arc branchial. Cette caractéristique correspond aux espèces qui se nourrissent et prélèvent leurs proies par filtration et généralement les proies planctoniques.

Toujours en ce qui concerne le régime alimentaire de ce poisson, précisons que les résultats obtenus par l'étude de l'indice alimentaire sont confirmés par la détermination de l'étude de l'intensité de 
prédation en fonction de la taille des spécimens de Labeobarbus altianalis (Figure 5). En effet, on note une diminution de l'intensité de prédation avec la taille du poisson. Les jeunes individus paraissent plus agiles dans la recherche et la capture des proies actives, d'autant plus que leur taille leur permet une meilleure prospection des microbiotopes existants. Ils se nourrissent essentiellement du phytoplancton, la classe de Bacillariophycées constitue l'aliment essentiel de Labeobarbus altianalis des affluents de la rivière Luhoho. Cette classe est incontestablement représentée par les genres Navicula, Nitschia et Tabellaria dont ce poisson s'en nourrit depuis $89,07 \mathrm{~mm}$ de longueur totale jusqu'à $268,00 \mathrm{~mm}$ de longueur totale. Ces genres sont accompagnés par les genres Monoraphidium, Gomphonema, Pinnularia, Surirlla, etc. Labeobarbus altianalis complète sa ration alimentaire avec les débris végétaux, les autres catégories des proies comme les insectes, le zooplancton s'avèrent supplémentaires et occasionnelles pour les crevettes et écaille pour les individus de petites tailles. Le changement de tendance alimentaire chez ce poisson au-delà d'une longueur de $89,07 \mathrm{~mm}$ se traduit par une réduction progressive de l'intensité de prédation.

De nos résultats, nous concluons que cette espèce est phytoplanctonophage. Cependant les espèces du genre Barbus auquel appartenait cette espèce ont des régimes très variés en fonction de l'âge du poisson. Les jeunes individus ont une tendance zoophage, dominé par les larves de Diptères et d'Ephéméroptères, les plus âgés par contre, présentent une tendance phytophage qui reflète l'opportunisme (Cherghou et al., 2002), et d'autres encore sont entomophages. Waasalo (2003) signale en effet qu'une espèce de Barbus à l'occurrence de B. kerstenii lac Kivu est entomophage avec un rythme d'activité trophique nocturne. Selon elle, cette espèce se nourrit aux dépens de larves d'insectes.

\section{Conclusion}

L'étude de l'écologie alimentaire de Labeobarbus altianalis (Boulenger, 1900) (Cyprinidae), par analyse qualitative et qualitative des contenus stomacaux, a porté sur 235 individus, de longueurs à la fourche comprise entre $89,00 \mathrm{~mm}$ et $268,90 \mathrm{~mm}$, provenant des rivières Tchinganda, Tchoko, Nyamunene et Eke dans le bassin de la rivière Luhoho de Bunyakiri en territoire de Kalehe (R.D.Congo), et capturés au filet épervier de $10 \mathrm{~mm}$ de maille d'entre-nœuds et de $2 \mathrm{~m}^{2} \mathrm{de}$ surface pendant la période de mars à juin 2014.Pendant cette étude, un cycle de 12heures de pêche a été réalisé. Il ressort de cette étude que Labeobarbus altianalis des affluents de la rivière Luhohose nourrit préférentiellement de phytoplancton des groupes des diatomées (Bacillariophycées), des Cyanophytes, des Chlorophytes et d'Euglenophytes dans tous les sites considérés et à tous les âges. Cependant, il complète son régime par les débris végétaux, le zooplancton, les crevettes et les écailles. Son activité locomotrice serait plus intense entre 6heures et 18 heures à Tchinganda et Eke. Par contre, à Tchoko et Nyamunene, c'est entre 14heures et 18heures qu'il se déplace intensément. L'activité locomotrice de ce poisson serait liée à la recherche de la nourriture. Il existe un lien étroit entre l'activité locomotrice de ce poisson et son alimentation dans tous les quatre sites d'échantillonnage considérés.

\section{CONFLIT D'INTERETS}

Les auteurs déclarent qu'il n'y a aucun conflit d'intérêt.

\section{CONTRIBUTIONS DES AUTEURS}

GMO est intervenu dans toutes les phases de l'étude. Il s'agit de la conception de l'étude, la collecte des données, le dépouillement, le traitement et l'analyse des données et la rédaction du présent manuscrit ; DDCK est intervenu dans la conception de l'étude, le dépouillement, le traitement et 
l'analyse des données et la rédaction du présent manuscrit; VNM est intervenu dans la conception de l'étude, le traitement des données et dans la correction du manuscrit, JCM est intervenu dans la correction du manuscrit et ORR est intervenu dans la correction du manuscrit.

\section{REMERCIEMENTS}

Nos remerciements s'adressent à tous ceux qui, de près ou de loin, ont contribué à la réalisation de cette étude à savoir : les autorités politico-administratives $\mathrm{du}$ poste d'encadrement administratif de Bunyakirien général et le chef traditionnel en particulier pour leur accueil et soutien permanent sur le terrain ainsi qu'à tous les pêcheurs qui ont fortement contribués à la collecte des données indispensables à notre travail. Nous remercions également aux autorités du Centre de Recherche en Hydrobiologie d'Uvira (CRH/Uvira) pour nous avoir accueillis dans leur laboratoire où toutes les observations avaient été faite. A messieurs Solo et Ombeni, pêcheurs experts, qui nous ont assistés pendant les travaux de récolte des échantillons de ce travail.

\section{REFERENCES}

Bouleau G. 2007. La gestion française des rivières et ses indicateurs à l'épreuve de la directive cadre - CEMEGREF - Paris. $60 \mathrm{p}$.

Cherghou S, Khodari M, Yaakoubi F, Benabid M, Badri A. 2002 : Contribution à l'étude du régime alimentaire du barbeau (Barbus barbuscallensisValenciennes, 1842) d'un cours d'eau du Moyen-Atlas (Maroc): Oued Boufekrane. Rev. Sci. Eau, 15(1) : 153-163.

Cikwanine K. 2008. Inventaire systématique et écologie alimentaire de deux espèces d'Haplochromis (H. kamiranzovu et $H$. crebridens) du lac Kivu, bassins de Bukavu et Ishungu. Mémoire. D.E.A., Faculté des Sciences, Département de Biologie, Université du Burundi, 59 p.
Fernando CH. 2002. A guide to tropical freshware zooplankton, identification, ecology and impact on fishories; in Backhuys publishers, leiden, 291 pages.

Gangbe L, Agadjihouede H, Chikou A, Senouvo P, Mensah GA, Laleye P. 2016. Biologie et perspectives d'élevage de la crevette géante d'eau douce Macrobrachium vollenhovenii (Herklots, 1857). Int. J. Biol. Chem. Sci., 10(2): 573-598.

DOI

http://dx.doi.org/10.4314/ijbcs.v10i2.11

Hecky RE, Kling J. 1987. Phytoplankton ecology of the great lakes in the rift valleys of Central Africa. Arch. Hydrobiol. Beih. Ergebn. Limnol., 25: 197-228.

Hyslop EJ. 1980. Stomach contents analysisa review of methods and their application. J. Fish Biol., 19: 411-429.

Ibala ZA. 2010. Faune des poissons des rivières Luki et Lefini : Diversité et écologie. Thèse de Doctorat, K.U. L., PP1-100.

Isumbisho $\mathrm{M}$, Sarmento $\mathrm{H}$, Kaningini $\mathrm{B}$, Micha J-C, Descy J-P. 2006. Zooplankton of Lake Kivu, Eastern Africa, half a century after the Tanganyika sardine introduction. Journal of Plankton Research, 28(11): 971-989.

Kadjo V, Etchian OA, Blé CM, Soro D, Yapi JN, Otchoumou A. 2016. Caractérisation de la pêche aux crevettes d'eau douce Atyascabra (Leach, 1815) (Decapoda: Atydae) dans la rivière Bia, Côte d'Ivoire. Int. J. Biol. Chem. Sci., 10(2) : 620-631.

DOI

http://dx.doi.org/10.4314/ijbcs.v10i2.14

Kaningini M. 1995. Etude de la croissance, de la reproduction et de l'exploitation de Limnothrissa miodon du lac Kivu, bassin de Bukavu (Zaïre). Thèse de doctorat, Presses Universitaires de Namur, Belgique, 211p.

Khady Diouf G. 2015. Systématique des poissons, Formation de base FishBase et 
taxinomie des poissons, Session 2015, IFAN Ch. A. Diop, 45P.

Kisekelwa T. 2012. Contribution à la connaissance de l'ichtyofaune des rivières Lowa et Elila à l'Est de la RDC. Mémoire Master, Université du Burundi, $58 \mathrm{P}$.

Kisekelwa T, Isumbisho M, 2010. Composition qualitative du zooplankton en amont et en aval du barrage de Mururu. Cahier du CERUKI, Nouvelle Série, 40 : 28-32.

Kisekelwa $\mathrm{T}$, Isumbisho $\mathrm{M}$, Ntakimazi $\mathrm{G}$, Micha JC. 2013. Preliminary assessment of fish diversity in Lowa River. Bulletin Scientifique de l'Institut National pour l'Environnement et la Conservation de la Nature, 12: 17-24.

Laleye P, Chikou A, Philippart JC, Teugels G, Vandewalle P. 2004. Etude de la diversité ichtyologique du bassin du fleuve Ouémé au Bénin (Afrique de l'Ouest). Cybum, 28(4): 329-339.

Mamadou D, Ghorbel M. 2010. Etude du régime alimentaire de Pomadasys incisus (Haemulidae) des cotes de Nouadhibou (Mauritanie). Bull. Inst. Natn. Scien. Tech. Mer de Salammbô, 37: 31-41.
Mpawenayo B. 1996. Les eaux de la plaine de la Ruzizi (Burundi) : les milieux, la flore et la végétation algale. Thèse de doctorat, Académie Royale des Sciences d'Outre- Mer, Bruxelles, 236p.

Nshombo M, Lushombo M. 2010. The Introduction of the Endemic Fish Species, Lamprichthys tanganicanus (Poeciliidae), from Lake Tanganyika into Lake Kivu: Possible Causes and Effects. Aquatic Ecosystem Health \& Management, 13(2): 1-11.

Ramade F. 2003. Eléments d'Ecologie: Ecologie Fondamentale, Eaux Courantes/Systèmes Lotiques. Edition Dunod ; $688 \mathrm{p}$.

Ramade F. 2005. Eléments d'Ecologie: Ecologie Appliquée (6 ${ }^{\text {ème }}$ édn). Dunod; $833 \mathrm{p}$.

Zango P, Tomedi MTE, Efole TE, Tiogue CT, Nguenga D, Kamanke SM, Mikolasek, Tchoumboue J. 2016. Performances de reproduction du poisson chat endogène $\mathrm{du}$ Cameroun Clarias jaensis (Boulenger, 1909) en milieu contrôlé. Int. J. Biol. Chem. Sci., 10(2): 533-542. DOI

http://dx.doi.org/10.4314/ijbcs.v10i2.7 